South African Journal of Geomatics, Vol. 6. No. 2, Geomatics Indaba 2017 Special Edition, August 2017

\title{
An algorithm to retrieve Land Surface Temperature using Landsat-8 Dataset
}

\author{
Ayodeji Ogunode $^{1}$, Mulemwa Akombelwa ${ }^{1}$ \\ ${ }^{1}$ Programme of Land Surveying, University of KwaZulu-Natal, South Africa, ogunodeap@ gmail.com
}

DOI: http://dx.doi.org/10.4314/saig.v6i2.10

\begin{abstract}
Soil moisture, surface temperature, and vegetation are variables that play an important role in our environment which in turn increases the demand for accurate estimation of certain geophysical parameters such as weather, flooding, and land classification. However, for accurate Land Surface Temperature (LST) estimation, remotely sensed data of key environmental forms were considered and applied in this research. The goal of this study was to apply a suitable algorithm for LST estimation from the Landsat-8 dataset that gives a great accuracy when compared with in-situ observations.

Spatial and temporal Landsat-8 data were acquired which provided the analytical structure for linking specific data successfully due to fine resolutions. The data were then applied to determine brightness temperatures, vegetation cover, and surface emissivity which demonstrated the effectiveness of the Split-Window Algorithm as an optimum method for LST retrieval from satellite.

The results show temperature variation over a long period of time can be used in observing varying temperature values based on terrain i.e. High temperatures in fully built up areas and low temperatures in the well-vegetated regions. Finally, accurate LST estimation is important for land classification, energy budget estimations as well as agricultural production.
\end{abstract}

Keywords: Emissivity, Landsat, Land Surface Temperature, Split-Window, Vegetation.

\section{Introduction}

Remote Sensing which is the science of using instrument-based techniques in obtaining, analysis and interpreting data about objects or areas from a considerable distance without being in direct contact as against on-site observations (Lillesand et al., 2014). These techniques can vary from manual interpretation of aerial photos to the computer-based analysis of non-visible radiation collected by satellite-based sensors. Optical sensors can be broken down into three basic types: panchromatic, multispectral and hyperspectral. According to (Li et al., 2013, Qin and Karnieli, 1999), the extensive requirement of temperature data on a large scale for environmental studies and management activities of the Earth's resources by Environmentalists, Geologists and Health Practitioners has made the remote sensing of LST an important issue in recent decades and as such, many efforts have been devoted to the establishment of methodology for its retrieval. 
Remote sensing of LST using infrared radiation gives the average surface temperature of the scene covered by the radiometer's Field Of View (FOV). The spatial variation in LST acquisition causes a decrease in a measurement accuracy which in turn makes it difficult to validate LST from space using in-situ measurements (Minnett and Barton, 2010). To, therefore, retrieve LST from satellite Thermal Infrared (TIR) data, factors such as radiometric and atmospheric calibrations, surface emissivity correction as well as the characterization of spatial variability over land cover for vegetation has to be quantified. These factors are dependent on atmospheric conditions and emissivity of the land surface materials. Therefore, the effects of both atmosphere and Emissivity must be corrected through an algorithm for accurate LST estimation (Sobrino and Raissouni, 2000).

According to Ustin et al., (2004), there is a growing awareness among environmental scientists that remote sensing can and must play a role in providing the data needed to assess ecosystems conditions and to monitor change at all spatial scales. Thus, acquiring LST from remotely sensed data becomes one of the significant factors in this study. As a key parameter of the surface energy budget, LST is directly related to surface energy fluxes and to the latent heat flux, evapotranspiration and water stress Torrion et al., (2014), surface longwave emission and computing soil moisture Cammalleri and Vogt, (2015) and for understanding meteorological and hydrological processes in a changing climate (Duan et al., 2014).

This paper attempts to apply a Split Window (SW) algorithm, which requires two essential parameters (emissivity and transmittance) by using the Landsat- 8 dataset. Keeping the accuracy of the LST estimate, we avoid the complicated expression of the algorithm and the difficult calculation of its parameters. A complete and detailed description is given to the derivation of the algorithm, which includes the theoretical basis for remote sensing of LST.

\section{Review of Landsat for LST Retrieval}

The Landsat Programme, launched in the early 1970s had its capacity innovatively expanded over time which in turn increased the quality and size of captured data by onboard sensors. So far, there have been eight Landsat satellites launched successfully with the commencement of the Landsat-1 in 1972. The Landsat satellites have a near-earth orbit operation, scanning a ground area with $705 \mathrm{~km}$ altitude. Landsat 1-3 with both the infrared and visible bands had an $80 \mathrm{~m}$ resolution for the $185 \times 185 \mathrm{~km}$ imagery. Landsat 4-5 were equipped with double multispectral sensors; Thematic Mapper and Multispectral Scanner, with seven high-resolution spectral bands. Landsat 6 encountered a failure due to its immediate loss after launching while the Landsat $7 \mathrm{ETM}^{+}$was simply an advanced variant of the TM with a panchromatic band with high resolutions. Landsat- 8 has two major instruments (TIRS and OLI), at the 30m spatial resolution for the SWIR, visible and NIR; 100m for the TIR and $15 \mathrm{~m}$ for the panchromatic bands (Markham et al., 2013). The presence of double TIR bands in the Landsat-8 OLI/TIRS satellite in the atmospheric window $(10-12 \mu m)$ is the main advantage over previous Landsat series. 
In the research by Mallick et al., (2008), the potential of remote sensing to study the urban morphology is presented by estimating the spatial distribution and intensities of geophysical parameters using Landsat 7 ETM+. Their results show that the satellite derived emissivity values are in the acceptable range and the NDVI and fractional vegetation cover are effective in deriving surface emissivity. Sobrino et al., (2004) used Landsat TM 5 data to compare the proposed algorithms of Jiménez- Muñoz and Sobrino, (2003) and Qin et al., (2001) for LST estimation. Their results showed RMSD values of $2 \mathrm{~K}$ and $0.9 \mathrm{~K}$ respectively when $9 \mathrm{x} 9$ pixel samples are considered. Using Landsat $\mathrm{TM} / \mathrm{ETM}^{+}$data with a radiative transfer model, Li et al., (2004) suggested that the average difference is $0.98^{\circ} \mathrm{C}$ for Landsat 7 and $1.47^{\circ} \mathrm{C}$ for Landsat 5 when compared. However, all these studies used data providing daily to half-hourly coverage. They are appropriate for operational monitoring, but their low spatial resolution limits their use for detailed spatial analysis of land cover changes and intensively fragmented areas.

\section{Split Window Algorithm}

LST estimation from satellite TIR radiometers has been achievable through different systems; Single-Channel (SC), SW and Dual-Angle (DA) algorithms. Various studies concentrate on the utilization of sensors onboard the polar-orbiting satellites on account of their high spatial resolutions (Pandya et al., 2014). Accurate LST estimation by utilizing the SC algorithm require high-quality air transmittance codes in estimating air quantities, Land Surface Emissivity data, precise air profile and topographic impacts Sobrino et al., (2004) while involving the inversion of the Radiative Transfer Equation as shown in Equation [1]. A substitute methodology utilized over ocean uses the differential climatic absorption of both adjoining channels at 10-12 $\mu \mathrm{m}$ in the SW algorithm initially developed by McMillin, (1975) which does not need air profile data at acquisition time. The SW algorithm predicts that LSE in the TIR regions of the 10-12 $\mu \mathrm{m}$ region is established (Atitar and Sobrino, 2009, Sobrino and Raissouni, 2000). The DA algorithm as proposed by Sobrino et al., (2004) consolidates both the emissivity at forward and nadir perspective as it incorporates just emissivity reliance with no unequivocal WV reliance. This method requires that estimations are made for a fundamentally lengthy path; if not, the calculation becomes uncertain.

$$
L_{\text {sensor }}=\tau \varepsilon L_{T_{s}}+L_{u}+\tau(1-\varepsilon) L_{d}
$$

where $L_{\text {sensor }}$ is the radiance registered by the sensor, also referred to as Top of Atmosphere (TOA) radiance, $L_{T_{S}}$ is the black body radiance related to the surface temperature by Planck's law and $T_{S}$ is the LST, $L_{u}$ and $L_{d}$ are the upwelling and down-welling atmospheric radiances respectively in $\mathrm{Wsr}^{-}$ ${ }^{1} m^{-2} \mu m^{-1}, \tau$ is the atmospheric transmissivity and $\varepsilon$ is the LSE. The selection of the LST algorithm for Landsat-8 data is based on a literature review from Markham et al., (2013) and Tang and Li, (2014) and the characteristics of the Landsat- 8 satellite data. The single-window method requires a highquality atmospheric profile and is sensitive to uncertainties in the atmospheric corrections. Considering the simultaneous retrieval of the LST and the LSE, the Temperature and Emissivity Separation (TES) method may be a candidate. However, significant errors in the LST and LSE for 
the surfaces with low spectral contrast emissivity such as water, snow, vegetation can be caused by the TES (Gillespie et al., 2011). Since the Landsat-8 satellite observes the land almost at-nadir, the dual-angle algorithm was discarded. Among the various methods proposed for LST determination, the quadratic SW algorithm as shown in Equation [2] from Coll et al., (1994) and Sobrino et al., (1993) has received considerable attention because of its simplicity.

$$
T_{s}=T_{i}+A\left(T_{i}-T_{j}\right)^{2}+B\left(T_{i}-T_{j}\right)+C
$$

Where $T_{s}$ is the LST, $T_{i}$ and $T_{j}$ are the at-sensor brightness temperatures for two TIR channels, $A$ and $B$ are coefficients and $C$ is a constant. To make Equation [2] applicable, various research has been carried out. Such include Sobrino and Raissouni, (2000) that proposed modifying constant $C$ as the linear combination of $\varepsilon, \Delta \varepsilon$ and atmospheric water vapour content. François and Ottlé, (1996) presented different coefficients for different $\varepsilon$ values. Sun and Pinker, (2003) addressed the SW coefficients according to different surface types to account for LSE effect. However, it is observed that the constant $C$ is a combination of the Mean:

$$
\text { Mean, } \left.\quad \varepsilon=\left(\varepsilon_{i}+\varepsilon_{j}\right) / 2\right)
$$

and difference;

$$
\Delta \varepsilon=\left(\varepsilon_{i}+\varepsilon_{j}\right)
$$

from the LSE of the TIRs channels, thereby keeping the other coefficients independent of LSE. It should be noted that the land surface is complex and that the LSE may be quite different from unity and depends on the channel as stated in (Coll and Caselles, 1997). Considering the effect of the emissivity, the quadratic SW algorithm may not work well. As François and Ottlé, (1996) noted in their work, when $\varepsilon$ is greater than 0.95 , good accuracy can be obtained using the quadratic method with the emissivity-dependent coefficients. That means, when $\varepsilon$ is low, the quadratic relationship no longer performs well or is changed. Therefore, the quadratic method has to be re-examined closely, especially for low emissivity.

An efficient and convenient method for LST retrieval must be explored and investigated as it is influenced by numerous elements such as surface layout, land cover, and aerosol. Though different SWAs such as Jimenez-Munoz et al., (2014), Rozenstein et al., (2014) and Yu et al., (2014) for LST retrieval theoretically has been produced, it remains a complex procedure to get from satellite imagery. A systematic and direct framework is, therefore, important to streamline the operating procedures which are shown in Figure [1]. For this research, an imagery processing framework utilizing the Spatial Model Maker and ATCOR module of ERDAS Imagine in deriving LST specifically from Landsat-8 dataset was applied, which can also be used for determining Urban Heat Island (UHI) through LST estimation. Radiometric and geometric rectifications were done on the inputting imagery to ensure comparative imagery have the same resolutions. A simplified LST computation directly with this model is described in Qin and Karnieli, (1999) which shows the detailed processes. 
Using Equation [1], $\varepsilon$ and $\Delta \varepsilon$ can be examined in terms of $T_{i}-T_{j}$. For this reason, the Top of Atmosphere in the Landsat- 8 thermal bands $T_{10}$ and $T_{12}$ (where 10 and 12 represent respective wavelengths) were then simulated. By assuming that $\varepsilon_{10}=\varepsilon_{12}=1$, Equation [2] therefore gives a result of $0.29(A), 1.45(B)$ and 0.17(C) with a 0.67 RMSE (Coll et al., 1994, Sobrino et al., 1993). This result is further verified from Jin et al., (2015) which shows that SWA coefficients for Sea Surface Temperature estimation can be utilized for LST retrieval if there is an estimation of the $\varepsilon$ effect. A modified $\mathrm{C}$ is therefore combined with the results of $\mathrm{A}$ and $\mathrm{B}$ in developing the LST algorithm.

A major factor to be considered is, however, the ambient temperature of the study area through the Atmospheric Transmittance (AT). This is important as the saturated water vapour content affects the satellite imagery in the form of cloud cover. Hence, an estimation of the AT values becomes a necessity. To derive the AT, two main calculations are done namely Water Vapour Content and Mean Atmospheric Temperature. Relative Humidity ( $\mathrm{RH})$, as well as the temperature of upper-air estimation, are two fundamental estimations utilized as a part of the numerical climate model analysis for operational climatic forecasts. Equations [3] - [6] as described in (Jarraud, 2008, McRae, 1980, Wagner and Pruß, 2002) were applied in showing the relationship between saturation water vapour pressure and water vapour at a specific temperature.

$$
\begin{aligned}
& e_{w}(p, t)=f(p) * e_{w}(t) \\
& f(p)=1.0016+\left(3.15 * 10^{-6} * p\right)-\left(0.074 * p^{-1}\right) \\
& e_{w}(t)=6.112 \operatorname{Exp}[(17.62 * t) /(243.12+t)] \\
& W=10^{4} * R H *\left(\frac{e_{w}(p, t)}{P_{a}}\right)
\end{aligned}
$$

Where $e_{w}(p, t)$ is the saturation vapour pressure of moist air; $f(p)$ is the pressure function; $e_{w}(t)$ is the saturation vapour pressure in pure phase; $t$ is the ambient temperature; $p$ is the atmospheric pressure; $W$ is the water vapour content and $P_{a}$ is the standard atmospheric pressure of $1013.25 h P_{a}$. 


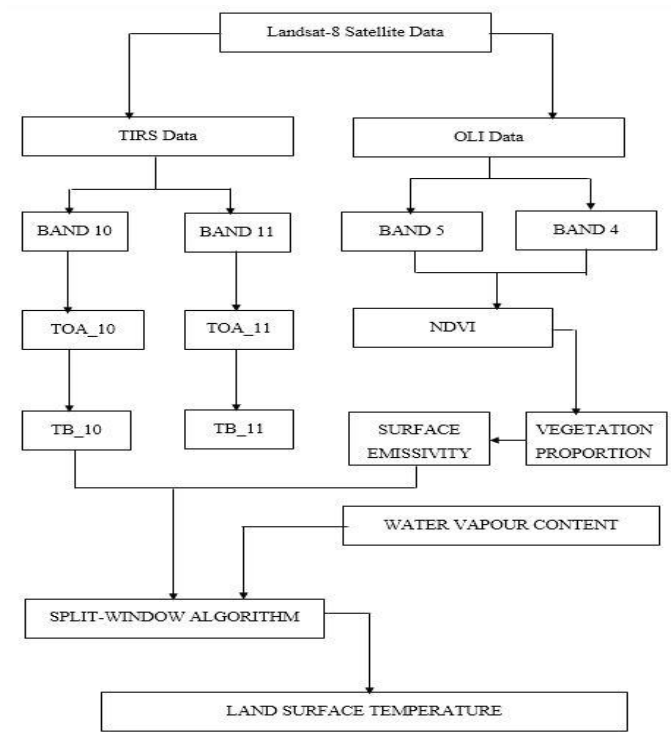

Figure 1: LST retrieval flowchart

\section{Application of algorithm}

For the application and validation of the SWA, Landsat-8 data were downloaded from EarthExplorer as well as observational data (atmospheric pressure, relative humidity, and temperature) were obtained from the South African Weather Station (SAWS) for the corresponding dates for which the satellite imagery was acquired. Data from the satellite imagery were radiometrically normalized, converted to surface reflectance, and quantitatively analysed to enable the establishment of generic and consistent classification rules after which the main processing began accurate LST retrieval through the SWA.

As shown in Figure [1], the following processing was then done in deriving the LST:

\subsection{TOA spectral transmittance}

The purpose of this was to calibrate the produced noise by the sensors which measure reflectance from the Earth surface in the form of Digital Numbers (DN) representing each pixel. The calibration as used by (Vicente-Serrano et al., 2008) was implemented for the TIR bands as shown in Equation [7]:

$$
L_{\lambda}=\left(\frac{L_{\max }-L_{\min }}{D N_{\max }}\right) * \text { Band }+L_{\min }
$$

Where $L_{\min }$ and $L_{\max }$ are spectral radiances, $D N_{\max }$ is the maximum DN and $L_{\lambda}$ is the TOA.

\subsection{Brightness Temperature}

After the conversion of DNs to reflection, the TIR data were then converted to Brightness Temperatures $\left(T_{B}\right)$ which "is the microwave radiance traveling upward from the top of Earth's atmosphere" Yang et al., (2014) using the thermal constants provided in the metadata file. In order to achieve accuracy for the $T_{B}$ conversion, equation [8] was implemented through the ATCOR module of ERDAS Imagine. The result is converted to Celsius from Kelvin by adding the absolute zero. 
South African Journal of Geomatics, Vol. 6. No. 2, Geomatics Indaba 2017 Special Edition, August 2017

$$
T_{B}=\frac{K_{2}}{\ln \left(\frac{K_{1}}{L_{\lambda}}+1\right)}
$$

Where $K_{1}$ and $K_{2}$ are band-specific constants

\subsection{Normalized Difference Vegetation Index}

The NDVI is an indicator used to analyse the biomass or greenness of the observed area. As Weng et al., (2014) stated, estimating NDVI is essential since the amount of vegetation present is a factor for LST retrieval. Equation [9] was used in deriving the NDVI.

$$
N D V I=\left(\frac{N I R(\text { Band } 5)-\operatorname{Red}(\text { Band } 4)}{N I R(B a n d 5)+\operatorname{Red}(\text { Band } 4)}\right)
$$

\subsection{Fractional Vegetation Cover}

Spatial variation in the radiometric temperature of surfaces is related to variations of the soil-water concentration vertically and surface greenness detailed by the FVC. The variations of detailed explanations of FVC utilized by remote sensing and modeling, be that as it may, can prompt an error between what is utilized as part of a model and what is estimated utilizing remote sensing. Hence, the formula as described in Wang et al., (2015) was used in deriving the FVC from the NDVI image as shown in Equation [10].

$$
F V C=\left(N D V I-N D V I_{\min } / N D V I_{\max }-N D V I_{\min }\right)^{2}
$$

\subsection{Land Surface Emissivity}

The LSE must be known in order to estimate LST since it is the relative capacity of a surface to discharge radiative energy and characterized as the proportion of black-body radiative energy (Planck's law) to land surface radiative energy of similar temperatures (Jimenez-Munoz et al., 2014). Nonetheless, it stays a challenge to absorb satellite radiance within the TIR regions $(10-12 \mu \mathrm{m})$ because of the absence of infrared LSE. Fortunately, several methods in remote sensing have been proposed for LSE retrieval. According to (Dutta, 2015), LSE can be calculated using:

$$
L S E=\varepsilon_{S} *(1-F V C)+\left(\varepsilon_{V} * F V C\right)
$$

Where, $\varepsilon_{s}$ and $\varepsilon_{V}$ are the Soil and Vegetation Emissivity constants for TIR bands respectively as shown in Table [1].

Table 1: Emissivity constants (Jimenez-Munoz et al., 2014)

\begin{tabular}{|c|c|c|}
\hline Emissivity & Band 10 & Band 11 \\
\hline$\varepsilon_{S}$ & 0.971 & 0.977 \\
\hline$\varepsilon_{V}$ & 0.987 & 0.989 \\
\hline
\end{tabular}




\subsection{Mean and Difference LSE}

Over a short distance, there can be a substantial LSE change. The possibility of utilizing the NDVI values for the retrieval of the vegetation and soil proportion; or the minimum and maximum NDVI value; so as to be able to estimate LSE and in turn the mean and difference LSE as required in the SWA application. The mean and difference LSE shows the TIR bands relationship with the applied formula of:

$$
\begin{aligned}
& \operatorname{Mean}(\varepsilon)=\frac{L S E_{10}+L S E_{11}}{2} \\
& \operatorname{Difference}(\Delta \varepsilon)=L S E_{10}-L S E_{11}
\end{aligned}
$$

\subsection{Atmospheric Transmittance}

Atmospheric transmittance which is characterized as atmospheric capacity in transmitting electromagnetic energy is reliant on air masses by radiation, water vapour, and air dust. It fluctuates for radiation of various wavelengths; smaller light scattering and atmospheric absorption, greater air transmittance. The value of $\mathrm{W}$ as shown in Equation (6) is incorporated for the AT when using the SW Algorithm needed in the LST retrieval process.

\subsection{Land Surface Temperature}

SWA utilizes both TIR bands normally situated in the $10-12 \mu \mathrm{m}$ atmospheric window. According to (Jiménez-Muñoz, 2008, Kamran et al., 2015), "the basis of the SW algorithm is that the radiance attenuation for atmospheric absorption is proportional to the radiance difference of simultaneous measurements at two different wavelengths, each subject to different amounts of atmospheric absorption". Therefore, processes $4.1-4.7$ is combined with the LST retrieval process as described in Li et al., (2013) and Rozenstein et al., (2014) to give Equation [14].

$$
\begin{gathered}
L S T=T_{B_{10}}+C_{1}\left(T_{B_{10}}-T_{B_{11}}\right)+C_{2}\left(T_{B_{10}}-T_{B_{11}}\right)^{2}+C_{0}+\left(C_{3}+C_{4} W\right)(1-\varepsilon)+ \\
\left(C_{5}+C_{6} W\right) \Delta \varepsilon
\end{gathered}
$$

\section{RESULTS AND DISCUSSION}

As described in the processing, the results entailed spatial distribution maps showing the NDVI, FVC and LSE values. LSE was created using the NDVI threshold technique where the NDVI was reclassified into soil and vegetation. Highly elevated regions had more vegetative cover; hence, LSE was high in these regions. Figure [2] has been derived using LSE, $T_{B}$ and Emissivity difference between band 10 and 11 .

An analysis of Figure [2] shows region (a) having highest temperatures of $22-28^{\circ} \mathrm{C}$, (b) having mixed temperature levels due to the dam presence and low inhabitants, (c) is well populated with average temperatures of $18-23^{\circ} \mathrm{C}$, (d) which is the busy Durban central business district having varying temperatures between $13-23^{\circ} \mathrm{C}$ while (e) and (f) have relatively low temperatures due to being along the coastline with low inhabitants. The long variation in the temperature values is attributed to taking the mean temperatures over the time stamp covering all seasons. Also, the results were slightly 
affected due to errors as indicated in the analysis section for the time series was acquired from the satellite. Input parameters when estimating RH and $W$ contributed to slight errors where some regions had either lower or higher temperatures.

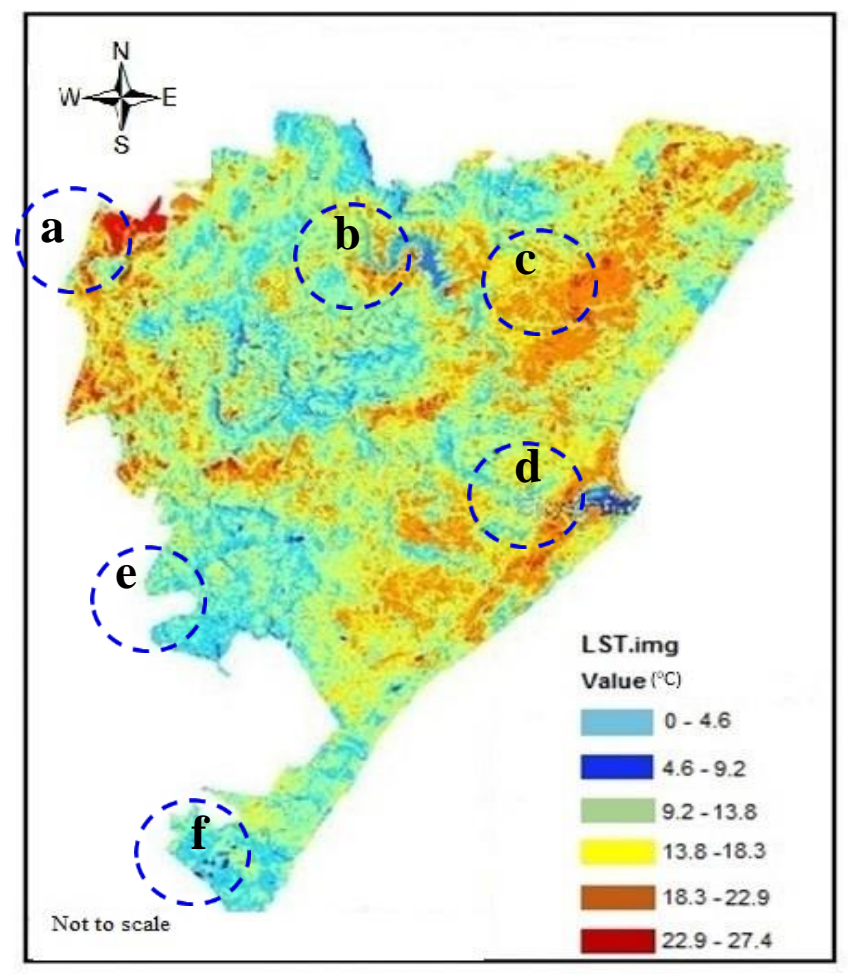

Figure 2: Durban LST map showing min-max values (June 2014 - May 2015)

From the above results, it can be observed that the UHI effect is a reality over the city centre which is along the harbour as well as suburban, industrial and highly dense unplanned areas i.e. natural processes and human activities, influence LST. The process was then repeated in two other study areas (East London and Polokwane) which have a slightly different climatic condition from Durban for validation purposes.

LST is not only influenced by land-use/land-cover types, but also by elevation, vegetation coverage, local meteorological conditions, and landscape composition. Therefore, even for the same land-use type, the mean LST may be different in different locations along the urban-rural gradient. In this study, the simple linear regression was conducted to evaluate the relationships between LST and elevation derived from Digital Elevation Model (DEM) to explore the relationship between LST and vegetation fraction (NDVI). 


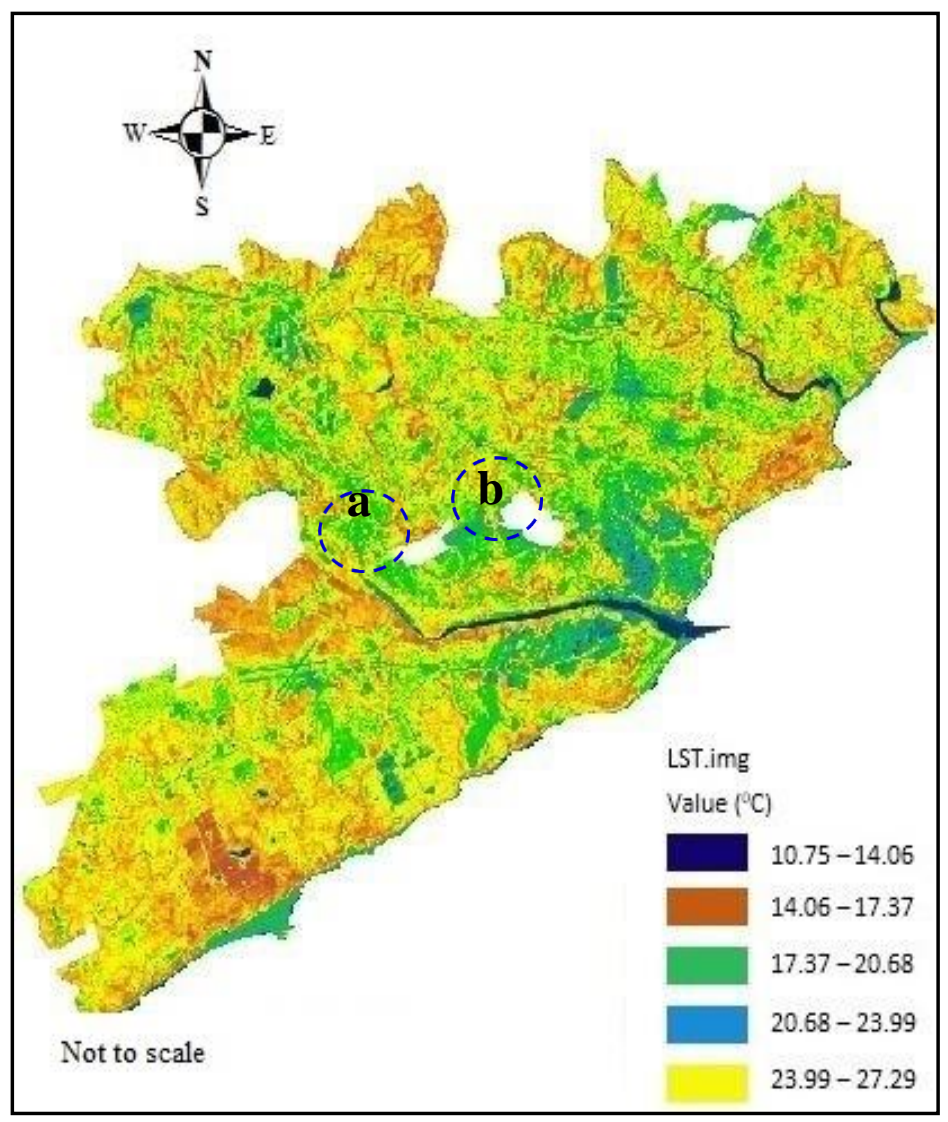

Figure 3: East London LST map showing min-max values (June 2014 - May 2015)

The white blanks regions (a) and (b) of Figure [3] was as a result of the shapefile obtained from the Eastern Cape municipality which was used in clipping from the raw downloaded Landsat-8 data. Figures [3] and [4] which were the validation sites showed higher LST in urban areas when compared to the non-urban areas.

In order to reveal the diversities of LST in different types of Landcover, the temperature characteristics of four Landcover types were analysed. The minimum, maximum and mean temperatures of the land cover patterns were derived by averaging all corresponding pixel values. The preceding results reflect that the spatial variability of LST is basically consistent with Landcover types of the study sites. In summary, the results had a wide range due to the range of temperatures being considered over various seasons.

\section{Analysis}

Sensitivity, statistical regression and error analysis on each study site was then carried out to further determine the validity of this SWA and determine error effects from estimations. 


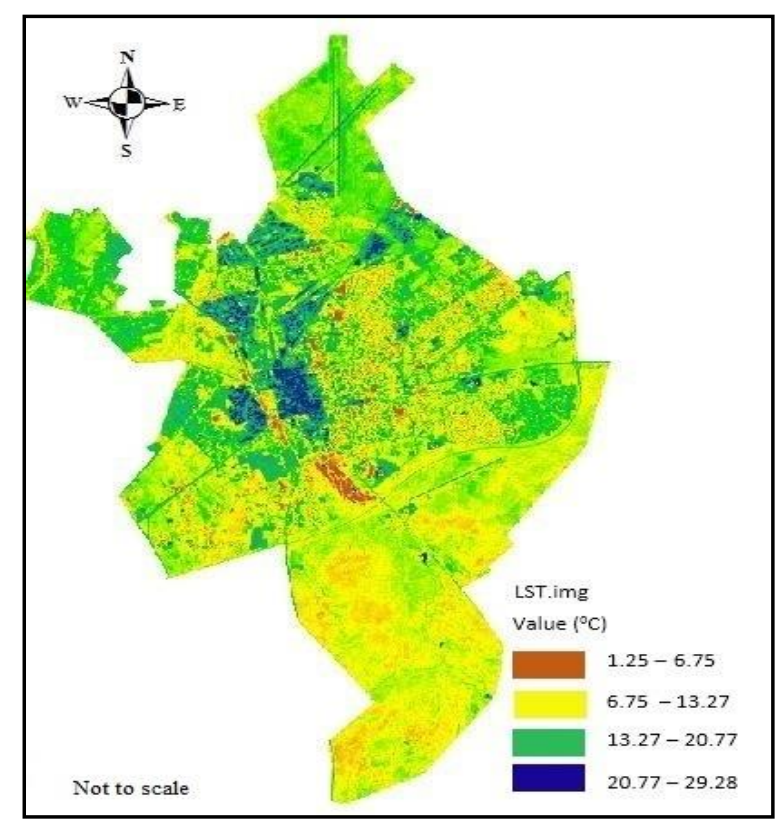

Figure 4: Polokwane LST map showing min-max values (June 2014 - May 2015)

\subsection{Sensitivity Analysis}

The sensitivity analysis provides possible error effects of the estimation parameters used for LST retrieval. LSE, at-sensor $T_{B}$ and AT were required for the application of this SWA and errors on these parameters subsequently led to LST errors. The analysis was performed using the python scripts as established by Isaya Ndossi and Avdan, (2016) in which various contributions to final LST error is estimated from numerous derivatives according to the classical error theory (Jin et al., 2015, Rozenstein et al., 2014). LST estimation errors are independent of change in temperatures as it changes over $0-60^{\circ} \mathrm{C}$ range by less than $\pm 0.03^{\circ} \mathrm{C}$ as $T_{10}-T_{11}=-2.2^{\circ} \mathrm{C}$, Table [2] and $0.1 \mathrm{gcm}^{-2}$ Atmospheric Water Vapour Content under-estimation. This little change, therefore, becomes negligible practically.

Table 2: Estimated LSE from NDVI and FVC

\begin{tabular}{|l|c|c|c|}
\hline Class & FVC & NDVI & LSE \\
\hline Water body & 0.013 & -0.171 & 0.989 \\
\hline Vegetation & 0.977 & 0.615 & 0.972 \\
\hline Built-up & 0.153 & 0.106 & 0.911 \\
\hline Bare soil & 0.029 & 0.026 & 0.895 \\
\hline
\end{tabular}

There is an error increase in LST estimations when the AWVC decreases which in turn causes an increase in the AT. The impact increases as $T_{B}$ difference between the increasing TIRS bands.

\subsection{Linear regression analysis}

Statistical regression analysis was used to establish a relationship between the estimated LST values and the measured air temperature values from the SAWS and was achieved by using mean temperature values over the study period (June 2014 to May 2015). $\boldsymbol{R}^{2}$ was achieved by utilizing Microsoft Excel and regression model formula of

$$
\gamma \approx f(x, \beta)
$$


where $\gamma$ is the dependent variable (measured LST) and $x$ is the independent variable (estimated LST). The analysis resulted in an $\mathrm{R}^{2}$ of 0.952 (Durban), 0.983 (East London) and 0.979 (Polokwane) as shown in Figure [7].

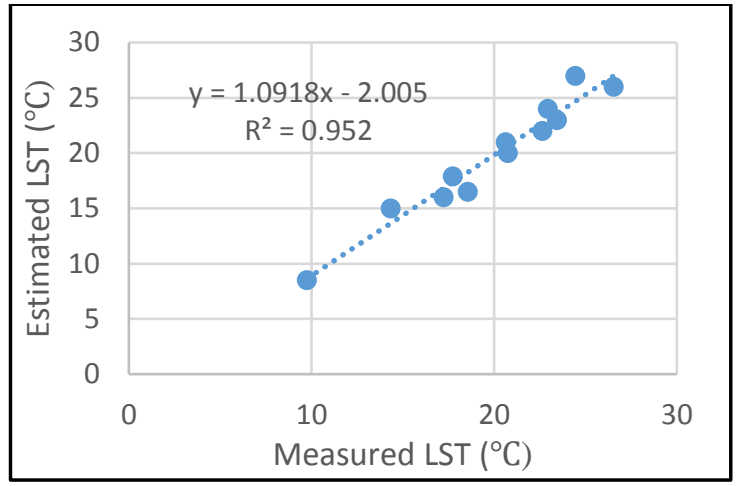

Figure 7: Linear regression analysis for Durban LST (June 2014 - May 2015)

\subsection{Error analysis}

Error from the split window algorithm used for LST retrieval is hard to measure. However, such errors are minimized.

Table 3: RMSE for Durban (June 2014 - May 2015)

\begin{tabular}{|c|c|c|r|c|}
\hline Month & Estimated $\left({ }^{\circ} \mathrm{C}\right)$ & Measured $\left({ }^{\circ} \mathrm{C}\right)$ & $\mathbf{R}^{2}$ & RMSE \\
\hline Jun & 9.75 & 8.5 & 1.56 & 1.19 \\
\hline July & 14.34 & 15 & 0.44 & \\
\hline Aug & 17.74 & 17.9 & 0.03 & \\
\hline Sept & 22.65 & 22 & 0.43 & \\
\hline Oct & 17.23 & 16 & 1.51 & \\
\hline Nov & 20.64 & 21 & 0.13 & \\
\hline Dec & 23.44 & 23 & 0.19 & \\
\hline Jan & 26.54 & 26 & 0.29 & \\
\hline Feb & 22.94 & 24 & 1.12 & \\
\hline Mar & 24.44 & 27 & 6.55 & \\
\hline Apr & 20.75 & 20 & 0.75 & \\
\hline May & 18.55 & 16.5 & 4.2 & \\
\hline
\end{tabular}


For the error with calibration, it showed that the results are within the pre-determined $\pm 0.6 \mathrm{~K}$ obtained after July 2014 to (http://landsat.usgs.gov/l8handbook_appendixa.php). Error from LSE can originate from the error of estimating NDVI and approximations. However, soil emissivity is highly relative within the study area which is well vegetated for a $\sim 0.979$ emissivity applicability. This research had below 0.005 emissivity error which prompted a $0.16^{\circ} \mathrm{C}$ maximum LST error (when interpolated with SAWS data). Thus, the overall estimate is $1.04 \mathrm{~K}-1.27 \mathrm{~K}$ RMSE in the LST estimation for the three study site as shown in Table [3].

Table [3] was then repeated for the other study sites - East London and Polokwane - with RMSE values of 1.27 and 1.04 respectively.

\section{Conclusion}

The applied SWA is a dynamic mathematical tool that provides LST information using the $T_{B}$ of TIRS bands, LSE derived from FVC of the OLI bands in accurately estimating LST with the addition of Atmospheric Transmittance. The applicability of this SWA from the results of validation and RMSE for the retrieved LSTs is confirmed when validated against observed SAWS data from the three study sites. On the basis of the validation and the sensitivity analysis, it can be concluded that the SWA presented in this paper is able to provide an alternative to ground-truth observations for accurate LST retrieval from the Landsat- 8 dataset.

\section{Acknowledgement}

Appreciation goes to the University of KwaZulu-Natal for providing an enabling environment for this research. Thanks also go to SAWS and eThekwini Municipality for providing raw data needed for processing and validation.

\section{References}

Atitar, M. \& Sobrino, J. A. 2009. A split-window algorithm for estimating lst from Meteosat 9 data: test and comparison with in situ data and modis 1sts. Geoscience and remote sensing letters, IEEE, 6.

Cammalleri, C. \& Vogt, J. 2015. On the role of land surface temperature as proxy of soil moisture status for drought monitoring in Europe. Remote sensing, 7, 16849-16864.

Coll, C., Caselles, V., Sobrino, J. A. \& Valor, E. 1994. On the atmospheric dependence of the split-window equation for land surface temperature. Remote sensing, 15, 105-122.

Duan, S.-B., Li, Z.-L., Tang, B.-H., Wu, H. \& Tang, R. 2014. Generation of a time-consistent land surface temperature product from modis data. Remote sensing of environment, 140, 339-349.

Dutta, R. 2015. Remote sensing of energy fluxes and soil moisture content. Journal of spatial science, 60.

François, C. \& Ottlé, C. 1996. Atmospheric corrections in the thermal infrared: global and water vapor dependent split-window algorithms-applications to atsr and avhrr data. IEEE transactions on geoscience and remote sensing, 34, 457-470.

Gillespie, A. R., Abbott, E. A., Gilson, L., Hulley, G., Jiménez-Muñoz, J.-C. \& Sobrino, J. A. 2011. Residual errors in aster temperature and emissivity standard products ast08 and ast05. Remote sensing of environment, 115, 3681-3694. 
Isaya Ndossi, M. \& Avdan, U. 2016. Application of open source coding technologies in the production of land surface temperature (lst) maps from Landsat: a pyqgis plugin. Remote sensing, 8, 413.

Jarraud, M. 2008. Guide to meteorological instruments and methods of observation (wmo-no. 8). World meteorological organisation: Geneva, Switzerland.

Jiménez-Muñoz, J.-C. 2008. Split-window coefficients for land surface temperature retrieval from lowresolution thermal infrared sensors. IEEE Geoscience and remote sensing letters, 5, 706-709.

Jimenez-Munoz, J. C., Sobrino, J. A., Skokovic, D., Mattar, C. \& Cristobal, J. 2014. Land surface temperature retrieval methods from Landsat- 8 thermal infrared sensor data. Geoscience and remote sensing letters, IEEE, 11, 1840-1843.

Jiménez- Muñoz, J. C. \& Sobrino, J. A. 2003. A generalized single- channel method for retrieving land surface temperature from remote sensing data. Journal of geophysical research: atmospheres, 108.

Jin, M., Li, J., Wang, C. \& Shang, R. 2015. A practical split-window algorithm for retrieving land surface temperature from Landsat- 8 data and a case study of an urban area in China. Remote sensing, 7, 43714390.

Li, F., Jackson, T. J., Kustas, W. P., Schmugge, T. J., French, A. N., Cosh, M. H. \& Bindlish, R. 2004. Deriving land surface temperature from Landsat 5 and 7 during smex02/smacex. Remote sensing of environment, 92, 521-534.

Li, Z.-L., Tang, B.-H., Wu, H., Ren, H., Yan, G., Wan, Z., Trigo, I. F. \& Sobrino, J. A. 2013. Satellite-derived land surface temperature: current status and perspectives. Remote sensing of environment, 131, 14-37.

Lillesand, T., Kiefer, R. W. \& Chipman, J. 2014. Remote sensing and image interpretation, John Wiley \& Sons.

Mallick, J., Kant, Y. \& Bharath, B. 2008. Estimation of land surface temperature over Delhi using Landsat-7 etm+. J. Ind. Geophys. Union, 12, 131-140.

Markham, B., Reuter, D., Montanaro, M., Lunsford, A., Barsi, J., Hook, S., Schott, J. \& Raqueno, N. 2013. Landsat-8 (formerly ldcm): mission update and thermal infrared sensor (tirs) initial post launch performance.

Mcmillin, L. M. 1975. Estimation of sea surface temperatures from two infrared window measurements with different absorption. Journal of geophysical research, 80.

Mcrae, G. J. 1980. A simple procedure for calculating atmospheric water vapor concentration. Journal of the air pollution control association, 30, 394-394.

Minnett, P. J. \& Barton, I. J. 2010. Remote sensing of the earth's surface temperature. Radiometric temperature measurements. Ii applications, 43, 333-391.

Pandya, M. R., Shah, D. B., Trivedi, H. J., Darji, N. P., Ramakrishnan, R., Panigrahy, S., Parihar, J. S. \& Kirankumar, A. 2014. Retrieval of land surface temperature from the Kalpana-1 vhrr data using a single-channel algorithm and its validation over western India. Isprs journal of photogrammetry and remote sensing, 94.

Qin, Z. \& Karnieli, A. 1999. Progress in the remote sensing of land surface temperature and ground emissivity using noaa-avhrr data. International journal of remote sensing, 20, 2367-2393.

Qin, Z., Karnieli, A. \& Berliner, P. 2001. A mono-window algorithm for retrieving land surface temperature from Landsat TM data and its application to the Israel-Egypt border region. International journal of remote sensing, 22, 3719-3746.

Rozenstein, O., Qin, Z., Derimian, Y. \& Karnieli, A. 2014. Derivation of land surface temperature for Landsat8 tirs using a split window algorithm. Sensors, 14, 5768-5780.

Sobrino, J. \& Raissouni, N. 2000. Toward remote sensing methods for land cover dynamic monitoring: application to Morocco. International journal of remote sensing, 21, 353-366.

Sobrino, J., Sòria, G. \& Prata, A. 2004. Surface temperature retrieval from along track scanning radiometer 2 data: algorithms and validation. Journal of geophysical research: atmospheres, 109.

Sun, D. \& Pinker, R. T. 2003. Estimation of land surface temperature from a geostationary operational environmental satellite (goes- 8). Journal of geophysical research: atmospheres, 108.

Tang, H. \& Li, Z.-L. 2014. Land surface temperature retrieval from thermal infrared data. Quantitative remote sensing in thermal infrared. Springer.

Torrion, J. A., Maas, S. J., Guo, W., Bordovsky, J. P. \& Cranmer, A. M. 2014. A three-dimensional index for characterizing crop water stress. Remote sensing, 6, 4025-4042.

Ustin, S. L., Roberts, D. A., Gamon, J. A., Asner, G. P. \& Green, R. O. 2004. Using imaging spectroscopy to study ecosystem processes and properties. Bioscience, 54, 523-534. 
South African Journal of Geomatics, Vol. 6. No. 2, Geomatics Indaba 2017 Special Edition, August 2017

Vicente-Serrano, S. M., Pérez-Cabello, F. \& Lasanta, T. 2008. Assessment of radiometric correction techniques in analyzing vegetation variability and change using time series of Landsat images. Remote sensing of environment, 112, 3916-3934.

Wagner, W. \& Pruß, A. 2002. The iapws formulation 1995 for the thermodynamic properties of ordinary water substance for general and scientific use. Journal of physical and chemical reference data, 31, 387535.

Wang, F., Qin, Z., Song, C., Tu, L., Karnieli, A. \& Zhao, S. 2015. An improved mono-window algorithm for land surface temperature retrieval from Landsat 8 thermal infrared sensor data. Remote sensing, 7, 4268-4289.

Weng, Q., Fu, P. \& Gao, F. 2014. Generating daily land surface temperature at Landsat resolution by fusing Landsat and modis data. Remote sensing of environment, 145.

Yang, L., Cao, Y., Zhu, X., Zeng, S., Yang, G., He, J. \& Yang, X. 2014. Land surface temperature retrieval for arid regions based on Landsat-8 tirs data: a case study in shihezi, northwest China. Journal of arid land, 6.

Yu, X., Guo, X. \& Wu, Z. 2014. Land surface temperature retrieval from Landsat 8 tirs - comparison between radiative transfer equation-based method, split window algorithm and single channel method. Remote sensing, 6, 9829-9852. 